\title{
Real world management of esophageal ulcers: analysis of their presentation, etiology, and outcomes
}

\author{
D. L. Cohen, A. Bermont, V. Richter, H. Shirin
}

The Gonczarowski Family Institute of Gastroenterology and Liver Diseases, Shamir (Assaf Harofeh) Medical Center, Zerifin, affiliated with the Sackler School of Medicine, Tel Aviv University, Israel.

\begin{abstract}
Background and study aims: Esophageal ulcers are a rare cause of upper gastrointestinal morbidity and may be due to different etiologies. We sought to systematically evaluate patients with esophageal ulcers and describe their presentations, endoscopic findings, etiologies, treatments, and outcomes.

Patients and methods: Patients diagnosed with esophageal ulcers over an 11-year period were retrospectively identified from our institution's electronic medical records.

Results: We identified 100 patients with esophageal ulcers (0.49\% of patients undergoing upper endoscopy). Half of them presented due to gastrointestinal bleeding and three-quarters were admitted to the hospital. The majority were in the lower esophagus. Twenty-two unique etiologies, including multiple iatrogenic causes, were diagnosed in 91 of the cases. The most common etiology was gastroesophageal reflux disease $(57 \%)$, followed by non-steroidal anti-inflammatory drug use $(7 \%)$, malignancies $(3 \%)$, vomiting $(3 \%)$, caustic ingestion (2\%), pill esophagitis $(2 \%)$ and radiation $(2 \%)$. Many etiologies showed a predilection for specific segments of the esophagus. Nine ulcers required endoscopic intervention and all were treated successfully. Repeat endoscopies were performed 5 times for diagnostic or "second look" reasons, none of which changed the patients' diagnosis or treatment. No patients required surgery or stricture dilation. One patient's ulcer was complicated by perforation and he subsequently died. Four other patients died from non-ulcer related causes.

Conclusions: While the majority of ulcers were due to gastroesophageal reflux disease, 22 different etiologies were identified. Many were due to medication or iatrogenic causes. Repeat endoscopy did not appear to be helpful. While the incidence was low, they were frequently associated with significant morbidity. (Acta gastroenterol. belg., 2021, 84, 417-422).
\end{abstract}

Keywords: Esophageal diseases, ulcer, epidemiology, peptic esophagitis

\section{Introduction}

Esophageal ulcers are an uncommon cause of upper gastrointestinal symptoms. In a study of 1140 patients with acute gastrointestinal bleeding, only 20 (2\%) were found to have esophageal ulcers as the cause (1). Additionally, esophageal ulcers do not represent a single entity as they may be due to various causes. The most common cause is gastroesophageal reflux disease (GERD) which was reported to be the etiology in 58 of $88(65.9 \%)$ esophageal ulcer cases in another study (2). While GERD is the most common cause, esophageal ulcers many be due to many other conditions such as medication use [non-steroidal anti-inflammatory drugs (NSAIDs) and antibiotics], radiation therapy, Crohn's disease, caustic ingestions, dermatological diseases, neoplasms, and infectious agents [candida, herpes simplex virus (HSV), cytomegalovirus (CMV), and human immunodeficiency virus (HIV)] $(1,2)$.

Given the various etiologies and relatively low prevalence, few studies have evaluated esophageal ulcers as a single entity. We therefore aimed to produce a systematic "real world" evaluation of patients presenting with esophageal ulcers at a single tertiary-referral medical center. We sought to define their presentation, endoscopic findings, the etiology of the ulcers, the treatment received, any complications from the ulcer, and their overall outcome.

\section{Methods}

A retrospective descriptive analysis of patients found to have esophageal ulcers on upper endoscopy was performed at Shamir (Assaf Harofeh) Medical Center. Adult patients (age 18 and older) who underwent endoscopy between March 2009 and March 2020 were identified based on ICD-9 code (530.2) via the medical center's electronic medical record system. An additional 50 endoscopy reports without the ICD-9 code of 530.2 were reviewed to ascertain whether cases of esophageal ulcer were not being properly coded. This review identified no missed cases of esophageal ulcers.

Demographic and clinical data was obtained via thorough chart review including reviewing the endoscopy and pathology reports, as well as any admission, discharge, consultation, and clinic notes. Esophageal ulcer was defined as a discrete break fully within the esophageal mucosa with a clearly circumscribed margin (2). A minimum size of $3 \mathrm{~mm}$ was required. If photodocumentation of the ulcer was performed, then the photographs within the endoscopy report were consulted to confirm the appropriateness of the ulcer being including in the study.

For patients who underwent multiple endoscopies revealing esophageal ulcers, only the first endoscopy (index case) was used in the study. The average number of ulcers was not calculated as many endoscopy reports

Correspondence to : Daniel L. Cohen, c/o The Gonczarowski Family Institute of Gastroenterology and Liver Diseases, Shamir (Assaf Harofeh) Medical Center, Zerifin, Israel 70300. Fax : +972-8-977-9727. Phone : +972-58-507-2773

E-mail : docdannycohen@yahoo.com

Submission date : 04/10/2020

Acceptance date : $21 / 12 / 2020$ 
did not list the exact number seen. For example, some reports described that "ulcers" were seen or that "a few ulcers" were noted, but did not specify an exact number. If multiple ulcers were seen, the size of the largest ulcer listed was used.

The etiology of the ulcer was determined based on clinical, endoscopic, and pathologic findings (2). For example, GERD was diagnosed as an etiology based on clinical findings such as heartburn or a documented history of GERD, as well as endoscopic findings such as esophagitis or a hiatal hernia. Pill-induced ulcers and NSAID-related ulcers were based on a clinical history consistent with those diagnoses, without any endoscopic or pathologic findings to suggest another etiology. Diagnoses requiring histological confirmation, such as infectious viral etiologies like CMV and HSV, were only diagnosed when confirmed on pathological evaluation. In general, particular attention was paid to the endoscopist's impression as stated in the endoscopy report. If the endoscopist determined that a specific etiology was the cause of the ulcer, this was assumed to be the case unless clear clinical or pathologic evidence contradicted that diagnosis. If a thorough evaluation of the chart was unable to definitively determine a diagnosis, then the ulcer was classified as "unknown etiology." All endoscopies were performed by either an experienced attending gastroenterologist or a fellow-in-training who was supervised by an attending physician during the endoscopy.

Statistical analysis was performed using IBM SPSS Statistics for Windows (IBM Corporation, Armonk, NY). The study was approved by the Institutional Review Board at our institution.

\section{Results}

\section{Study population}

A total of 148 potential cases were identified based on the ICD-9 code. After reviewing those potential cases, the cases that did not meet the criteria for esophageal ulcer were excluded (Figure 1). These were due to erosive esophagitis without a discrete ulcer, ulcers within the esophagogastric junction that involved the gastric cardia, and ulcerated lesions with a mass-like component.

After these exclusions, a total of 100 patients remained (53 men, 47 women) which constituted the study population. The average age was $69.0+/-18.4$ years with a range from 19 to 96 years old. The majority

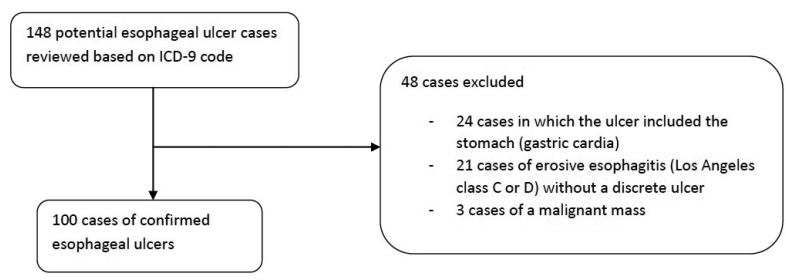

Figure 1. - Flowchart of esophageal ulcer cases.
Table 1. - Clinical Presentation $(\mathbf{n}=\mathbf{1 0 0})$

\begin{tabular}{|c|c|c|}
\hline \multicolumn{2}{|c|}{ Upper gastrointestinal bleeding } & 50 \\
\hline & hematemesis & 31 \\
\hline & melena & 27 \\
\hline \multicolumn{2}{|c|}{ Abdominal and/or chest pain } & 26 \\
\hline Dysphagia & & 21 \\
\hline Vomiting & & 15 \\
\hline Odynophagia & & 10 \\
\hline \multicolumn{2}{|c|}{ Anemia without gastrointestinal symptoms } & 8 \\
\hline Heartburn & & 5 \\
\hline Abnormal imaging study & & 3 \\
\hline Caustic ingestion & & 2 \\
\hline Food impaction & & 1 \\
\hline
\end{tabular}

(77 patients) were inpatients admitted to the hospital. Twenty-nine were taking aspirin or NSAIDs regularly.

These 100 patients with esophageal ulcers were diagnosed out of a total of 20,382 unique patients undergoing upper endoscopy at our institution during the study period, resulting in a prevalence of $0.49 \%$ (approximately 1 out of every 204 endoscopies). The 100 endoscopies were performed by 12 unique endoscopists.

\section{Presentation}

Half (50) of the patients presented due to gastrointestinal bleeding which was the most common reason. Of these, 31 had hematemesis and 27 had melena (some presented with both). Many patients presented due to multiple symptoms. Some of the other common presenting symptoms were abdominal and/or chest pain (26), dysphagia (21), vomiting (15), and odynophagia (10) (see Table 1).

\section{Endoscopic findings and ulcer characteristics}

Endoscopic findings are listed in Table 2. Solitary esophageal ulcers were identified in 66 patients while the remaining 34 patients had multiple esophageal ulcers. Ulcer size was described in 51 of the endoscopy reports. The average ulcer size was $15.5+/-13.0 \mathrm{~mm}$ with a range of 3 to $60 \mathrm{~mm}$. The most common location for ulcers was the lower-third of the esophagus (78) followed by the middle-third (18) and upper-third (11). This includes 5 patients who had ulcers in two parts of the esophagus and one who had ulcers throughout.

The vast majority of ulcers were clean-based (85), but 7 were noted to have active bleeding while another 8 had stigmata of recent bleeding, either an adherent clot or visible vessel. Fifteen patients had ulcers elsewhere in the upper GI tract (13 gastric ulcers, 2 duodenal ulcers). Endoscopy reports described hiatal hernias in 25 patients, while 24 described signs of reflux esophagitis. Candida was seen in 4 cases. Biopsies were taken from the ulcer for diagnostic purposes in almost half of the cases (48).

\section{Etiologies of the ulcers}

By means of thorough chart review, we were able to identify the etiology of the ulcer in 91 cases (Table 3). As expected, the most common cause was GERD which 
Table 2. - Endoscopic findings $(\mathbf{n}=\mathbf{1 0 0})$

\begin{tabular}{|l|c|c|}
\hline Multiple esophageal ulcers & & 34 \\
\hline Ulcer location & upper third & 11 \\
\hline & middle third & 18 \\
\hline Ulcer size (mm +/- SD, n=51) & lower third & 78 \\
\hline & minimum & 3 \\
\hline & maximum & 60 \\
\hline Signs of bleeding & & \\
\hline & actively bleeding & 7 \\
\hline & stigmata of recent & 8 \\
\hline & bleed & 85 \\
\hline Ulcers elsewhere in upper GI tract & clean ulcer base & \\
\hline \multicolumn{2}{|l|}{} & gastric ulcer \\
\hline Hiatal hernia & duodenal ulcer & 2 \\
\hline Reflux esophagitis & & 25 \\
\hline Esophageal candidasis & & 24 \\
\hline Prior surgery to the upper GI tract & & 4 \\
\hline Biopsies taken from the esophageal ulcer & 48 \\
\hline
\end{tabular}

$\mathrm{SD}$ : standard deviation. GI : gastrointestinal

accounted for 57 cases. Infectious etiologies included candida (2 cases), HSV (1), CMV (1), and idiopathic HIV ulcer (1). In 3 patients the ulcer was malignant (2 squamous cell cancers, 1 adenocarcinoma). Three patients also had ulcers felt to be related to prior vomiting and retching.

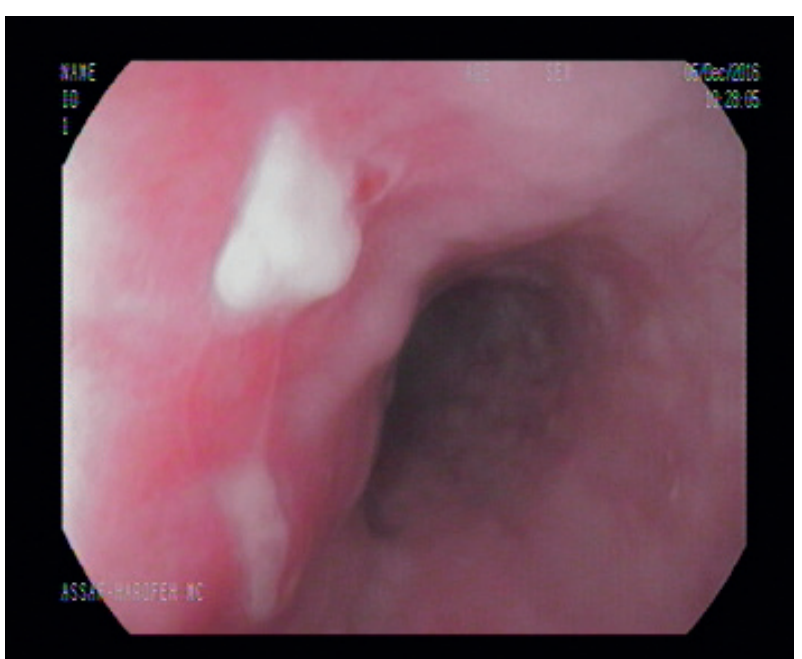

Figure 2. - A 57-year old woman was found to have ulcers in the mid-esophagus likely related to chronic NSAID use.

The etiology of the ulcer in numerous patients was iatrogenic or medication-related. These included 7 cases related to NSAID or aspirin use (Figure 2), 2 cases of pill esophagitis from antibiotic use (one patient was taking doxycycline, while the other was on both levofloxacin and amoxicillin-clavulanate), 2 cases due to radiation therapy given to treat lung cancer, 1 from nasogastric tube trauma, 1 from trauma from a recent endoscopic ultrasound, and 1 due to prior esophageal variceal band ligation. Other causes of ulcers included ingestion of a caustic substance (2 cases), Crohn's disease (1) (Figure 3),

Table 3. - Etiologies of the esophageal ulcers, including a breakdown of the location within the esophagus of each etiology

\begin{tabular}{|c|c|c|c|c|c|c|}
\hline \multirow{2}{*}{ GERD } & & \multirow{2}{*}{$\begin{array}{c}\text { overall } n=100 \\
57\end{array}$} & \multirow{2}{*}{$\begin{array}{c}\text { upper esophagus } \\
0(0)\end{array}$} & \multirow{2}{*}{$\begin{array}{c}\text { middle esophagus } \\
2(3.5)\end{array}$} & \multirow{2}{*}{$\begin{array}{c}\text { lower esophagus } \\
54(94.7)\end{array}$} & \multirow{2}{*}{$\begin{array}{c}\text { multiple locations } \\
1(1.8)\end{array}$} \\
\hline & & & & & & \\
\hline \multicolumn{2}{|c|}{ NSAID use } & 7 & $1(14.3)$ & $2(28.6)$ & $2(28.6)$ & $2(28.6)$ \\
\hline \multicolumn{2}{|c|}{ Malignancy } & 3 & $1(33.3)$ & $1(33.3)$ & $1(33.3)$ & $0(0)$ \\
\hline & Squamous cell cancer & 2 & $1(50.0)$ & $1(50.0)$ & $0(0)$ & $0(0)$ \\
\hline & Adenocarcinoma & 1 & $0(0)$ & $0(0)$ & $1(100)$ & $0(0)$ \\
\hline \multicolumn{2}{|c|}{ Vomiting/retching } & 3 & $0(0)$ & $1(33.3)$ & $2(66.7)$ & $0(0)$ \\
\hline \multicolumn{2}{|c|}{ Candida } & 2 & $2(100)$ & $0(0)$ & $0(0)$ & $0(0)$ \\
\hline \multicolumn{2}{|c|}{ Caustic ingestion } & 2 & $1(50.0)$ & $0(0)$ & $0(0)$ & $1(50.0)$ \\
\hline \multicolumn{2}{|c|}{ Pill esophagitis } & 2 & $1(50.0)$ & $0(0)$ & $1(50.0)$ & $0(0)$ \\
\hline \multicolumn{2}{|c|}{ Radiation therapy for lung cancer } & 2 & $0(0)$ & $2(100)$ & $0(0)$ & $0(0)$ \\
\hline \multicolumn{2}{|c|}{ Crohn's disease } & 1 & $0(0)$ & $0(0)$ & $0(0)$ & $1(100)$ \\
\hline \multicolumn{2}{|c|}{ Pemphigus } & 1 & $1(100)$ & $0(0)$ & $0(0)$ & $0(0)$ \\
\hline \multicolumn{2}{|c|}{ Prior variceal banding } & 1 & $0(0)$ & $0(0)$ & $1(100)$ & $0(0)$ \\
\hline \multicolumn{2}{|c|}{ Nasogastric tube trauma } & 1 & $0(0)$ & $1(100)$ & $0(0)$ & $0(0)$ \\
\hline \multicolumn{2}{|c|}{ Trauma from prior EUS } & 1 & $0(0)$ & $0(0)$ & $1(100)$ & $0(0)$ \\
\hline \multicolumn{2}{|c|}{ Marginal ulcer } & 1 & $0(0)$ & $0(0)$ & $1(100)$ & $0(0)$ \\
\hline \multicolumn{2}{|c|}{ Food impaction } & 1 & $1(100)$ & $0(0)$ & $0(0)$ & $0(0)$ \\
\hline \multicolumn{2}{|c|}{ Herpes simplex virus } & 1 & $0(0)$ & $0(0)$ & $1(100)$ & $0(0)$ \\
\hline \multicolumn{2}{|c|}{ Cytamegalovirus } & 1 & $0(0)$ & $0(0)$ & $1(100)$ & $0(0)$ \\
\hline \multicolumn{2}{|c|}{ Idiopathic HIV ulcer } & 1 & $1(100)$ & $0(0)$ & $0(0)$ & $0(0)$ \\
\hline \multicolumn{2}{|c|}{ Ischemia } & 1 & $0(0)$ & $0(0)$ & $0(0)$ & $1(100)$ \\
\hline \multicolumn{2}{|c|}{ Stasis esophagitis due to achalasia } & 1 & $0(0)$ & $0(0)$ & $1(100)$ & $0(0)$ \\
\hline \multicolumn{2}{|c|}{ Thermal injury from hot food ingestion } & 1 & $0(0)$ & $1(100)$ & $0(0)$ & $0(0)$ \\
\hline \multicolumn{2}{|c|}{ Unknown etiology } & 9 & $0(0)$ & $3(33.3)$ & $6(66.7)$ & $0(0)$ \\
\hline
\end{tabular}

GERD : gastrointestinal reflux disease. NSAID : non-steroidal anti-inflammatory drug. EUS : endoscopic ultrasound. HIV : human immunodeficiency virus. 


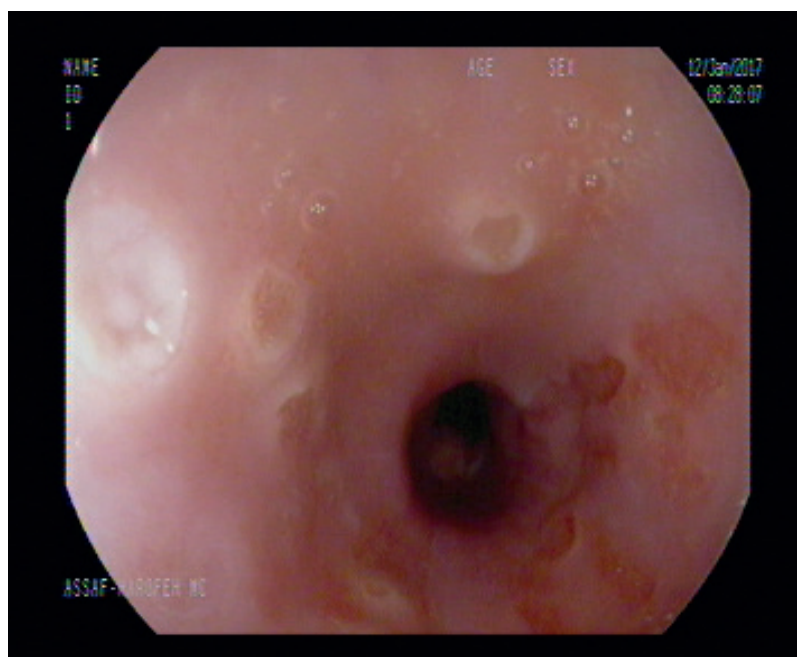

Figure 3. - A 21-year old woman was diagnosed with Crohn's disease of the ileum and right colon during a colonoscopy. On upper endoscopy, she was found to have multiple shallow circular ulcers throughout the esophagus due to Crohn's disease.

pemphigus (1), marginal ulcer (1), ischemia (1), prior food impaction (1), stasis esophagitis due to achalasia (1), and thermal injury due to swallowing hot food (1).

As expected, the etiologies of the ulcers differed by location within the esophagus (Table 3). For example, 54 of the 57 cases $(94.7 \%)$ of GERD ulcers were isolated to the lower-third of the esophagus. Other etiologies also showed a clear predilection to specific segments of the esophagus. Ulcers due to candida (2 cases), pemphigus (1), and food impaction (1) were only seen in the upper esophagus, while ulcers from radiation therapy (2) were only seen in the mid-esophagus. Other etiologies were more variable, such as NSAID use which was found in the upper esophagus (1), mid-esophagus (2), lower esophagus (2), and in multiple segments (2).

\section{Interventions}

Nine patients underwent endoscopic treatment of their ulcer. This included epinephrine injection in 7 cases and endoscopic clip placement in 5 ( 3 cases had both performed). All 9 of the treated ulcers were amongst the 15 ulcers that had either active bleeding or stigmata of recent bleeding (see Table 4). In all of these cases, the treatment was described as successful.

No patients required a repeat endoscopy due to rebleeding from the esophageal ulcer. However, repeat upper endoscopies were performed in 5 patients during their hospitalizations. Four times this was done with the intent of obtaining biopsies for diagnostic purposes as the ulcer was not sampled during the initial endoscopy. In the other case, a "second look" endoscopy was performed to assess the success of the endoscopic treatment performed during the initial endoscopy. These repeat endoscopies were performed between 2 days and 7 days after the initial one. In all cases, the ulcer was still seen on the repeat endoscopy. However, in none of these 5 cases
Table 4. - Interventions and complications $(\mathbf{n}=\mathbf{1 0 0})$

\begin{tabular}{|l|l|c|}
\hline \multicolumn{2}{|l|}{ Endoscopic treatment (overall) } & 9 \\
\hline & clip placement & 5 \\
\hline Blood transfusion & 7 \\
\hline Repeat inpatient endoscopy performed & 30 \\
\hline Follow-up outpatient endoscopy performed & 5 \\
\hline Surgery performed & 25 \\
\hline Endoscopic dilation performed & 0 \\
\hline CT scan performed & 0 \\
\hline Ulcer complications & 9 \\
\hline \multicolumn{2}{|l|}{ stricture } & 3 \\
\hline & perforation & 2 \\
\hline Death & (overall) & 1 \\
\hline & related to ulcer & 5 \\
\hline & from other causes & 1 \\
\hline
\end{tabular}

did the second endoscopy change the diagnosis or the treatment.

In 41 of the cases, the endoscopist recommended that a repeat endoscopy be performed as an outpatient. In 25 of those 41 cases $(61 \%)$, an outpatient endoscopy was performed at our hospital, ranging from 2 weeks to 4 months after the initial endoscopy. These cases revealed that the ulcer had completely healed in 23 of the 25 cases (92\%).

Thirty patients received blood transfusions, all of whom were inpatients. It was unclear how many of these patients required the transfusions specifically due to their esophageal ulcer as opposed to other causes of bleeding or anemia. Nearly all patients were given medical treatment with proton-pump inhibitors. No patients underwent a barium esophagram, either prior to or after their endoscopy. In 12 of the cases, the endoscopist recommended that a $\mathrm{CT}$ be performed, and the $\mathrm{CT}$ was actually performed 9 times (75\%). The indications for the CT scans were: to evaluate for a possible esophageal mass lesion (CT performed in all 5 cases; no masses noted); to evaluate the size of a hiatal hernia (performed in 2 of 3 cases); to evaluate for a possible perforation (performed in 2 of 2 cases; one case of perforation identified); and to evaluate for a possible extraesophageal lesion impinging on the esophagus (performed in 0 of 2 cases).

\section{Outcomes and Complications}

Only one patient had a perforation complicating his ulcer disease. This specific patient was extremely ill with sepsis after an intestinal resection due to ischemic bowel. It was decided to treat him conservatively (without surgery) due to his underlying critical illness, but his condition quickly worsened after the perforation was noted. He was the only patient where death was clearly related to the esophageal ulcer.

Four other patients died during their hospitalizations, but none were specifically related to the esophageal ulcer. The patients who died were: a patient with lung cancer who developed pneumonia and septic shock; a patient 
who presented with a cardiac arrest and developed hepatic failure; a patient who presented with urosepsis and developed renal failure; and a patient with urinary retention who experienced a cardiac arrest.

No patients underwent surgery due to their ulcers. Two patients were reported as having strictures at the site of the ulcer, but the endoscope was able to pass in both cases. No dilations were required.

\section{Discussion}

Few studies have specifically addressed esophageal ulcers, likely due to both the rarity of the diagnosis, as well as the large number of etiologies which can cause it. For example, some studies have focused on esophageal ulcers due to specific conditions, such as drug-induced ulcers $(3,4)$, or in specific populations, such as HIVinfected patients (5). This study reports on the features of 100 patients found to have esophageal ulcers and appears to be the largest cohort study to date evaluating real life experience with esophageal ulcers due to all causes.

Esophageal ulcers were diagnosed in $0.49 \%$ of patients undergoing upper endoscopy. This is a lower prevalence than has been reported elsewhere. Higuchi et al found the prevalence to be $1.2 \%$ (2), while older studies only evaluating patients with bleeding described it as the cause in $2 \%$ to $4 \%$ of cases $(1,6)$. These authors also discussed the use of barium esophagrams in the evaluation of esophageal ulcers (6). In our cohort none of the patients underwent this test. It appears to have been completely surpassed by endoscopy for the diagnosis and evaluation of esophageal ulcers.

We identified 22 unique etiologies of esophageal ulcers. Identifying the ulcer etiology is important to ensure that the patient receives proper therapy for the underlying cause. Similar to prior studies, we found that the majority of cases were due to $\operatorname{GERD}(1,2)$ Interestingly, we found that numerous ulcers were due to iatrogenic or medication-related causes. Similar to other studies, we found that NSAIDs were the second-leading cause of esophageal ulcers. However, we determined them to be the culprit in only $7 \%$ of cases, less than in other studies.For example, Higuchi et al found NSAIDs as the cause in $22.7 \%$ of cases (2) and Wolfson et al in $50 \%$ (1). Our numbers may undercount the true number of cases as complete medication lists were not always available.

As expected, we saw that many ulcer etiologies have a predilection for specific segments of the esophagus with candida, pemphigus, and food impaction causing upper esophageal ulcers, radiation therapy leading to midesophageal ulcers, and GERD causing distal esophageal ulcers. Interestingly, NSAIDs caused ulcers throughout the esophagus and included the middle third of the esophagus in 4 of 7 cases (57\%). This predilection for the mid-esophagus was also noted by Higuchi et al who found $80 \%$ of NSAID ulcers in the mid-esophagus (2), and Dağ et al who found $79 \%$ of drug-induced ulcers in the proximal or mid-esophagus (3).
Ulcer-related complications were rare in our cohort. Only two patients had stricturing at the ulcer site, neither of which required dilation to be performed. This is quite a contrast to Higuchi et al who performed endoscopic dilations in $12.5 \%$ of their cohort (2). These differences may be related to the easy availability of PPIs nowadays which have decreased the complications of NSAIDS and GERD.

None of our patients experienced re-bleeding from their esophageal ulcers requiring a repeat endoscopy. The 5 cases in which a repeat endoscopy was performed during their hospitalization were all for diagnostic or "second look" reasons. However, in none of those cases did the diagnosis or treatment of the patient change. Therefore, it appears that repeat inpatient endoscopies are not routinely needed. Additionally, the ulcer had healed in $92 \%$ of the follow-up outpatient endoscopies suggesting that routine endoscopic evaluation to confirm ulcer healing is also unnecessary.

Half of the patients presented due to gastrointestinal bleeding, and many were quite sick. Of the 77 inpatients, $30(39 \%)$ required blood transfusions. Some patients were admitted to the hospital with other critical illnesses when they developed esophageal ulcers, but, interestingly, we also noted that $23 \%$ of our cohort were found to have ulcers as part of an outpatient evaluation. This shows the diversity of clinical settings in which esophageal ulcers can be encountered.

Death occurred in 5 of our patients, but only in one was death directly related to the esophageal ulcer. Our ulcer-related death rate of $1 \%$ is less than that reported by Higuchi et al $(2.3 \%)$, as is our surgical rate $(0 \%$ vs $8.0 \%)$ (2). The largest analysis of death due to esophageal ulcers, a population-based study by Ranatanen et al, found 86 deaths out of 2,242 patients with esophageal ulcers (mortality rate of $3.8 \%$ ) (7). This is similar to our $5 \%$ all-cause mortality rate. These deaths are a reminder of the significant morbidity and mortality associated with esophageal ulcers.

The main limitation of this study is its retrospective nature. While we had access to each patient's electronic medical records from our institution, there was a wide range in the amount of data in each patient's chart. For example, some patients were referred for our hospital from an outside clinic for an outpatient endoscopic procedure. We therefore had fewer records available for such patients as compared to a patient who was admitted to our hospital. Hence, in some patients, we did not have access to all of the information that we desired including complete medical histories, comorbid conditions, alcohol and smoking histories, and medication lists. Further, we could not calculate measures of the patients' overall health such as the Charlson Comorbidity Index or the American Society of Anesthesiologists physical status classification.

Another limitation was our use of ICD codes to identify patients with esophageal ulcers. While this technique is commonly used in studies, it is possible that some 
patients with esophageal ulcers were not properly coded as such. If so, such patients would not have been included in our study and therefore our reported incidence rate may be lower than in reality. However, we did perform a review of 50 cases not coded as "esophageal ulcer" and found that none of them contained esophageal ulcers, suggesting that there is some accuracy to the coding.

Additionally, biopsies were only performed in 48 cases, and therefore pathological examination was limited. Some etiologies for ulcers that require biopsies may therefore have been undercounted. We also excluded cases of ulcerated masses and only included those that presented just with an ulcer. Therefore, we acknowledge that the number of malignancies in our study underrepresents the true number of esophageal malignancies diagnosed during the study period.

In conclusion, ulcer of the esophagus is a rare diagnosis which can be caused by numerous etiologies. Given the large number of causes, a thorough clinical history and evaluation is often required to make the correct diagnosis and treat properly. While the incidence of esophageal ulcers is low, they are often associated with significant morbidity and mortality suggesting that these patients require careful treatment and monitoring.

\section{References}

1. WOLFSEN HC, WANG KK. Etiology and course of acute bleeding esophageal ulcers. J. Clin. Gastroenterol., 1992 Jun, 14(4) : 342-6.

2. HIGUCHI D, SUGAWA C, SHAH SH, TOKIOKA S, LUCAS CE. Etiology, treatment, and outcome of esophageal ulcers: a 10-year experience in an urban emergency hospital. J. Gastrointest. Surg., 2003 Nov, 7(7) : 836-42.

3. DAG MS, OZTURK ZA, AKIN I, TUTAR E, CIKMAN O, GULSEN MT. Drug-induced esophageal ulcers: case series and the review of the literature. Turk. J. Gastroenterol., 2014 Apr, 25(2) : 180-4.

4. SUGAWA C, TAKEKUMA Y, LUCAS CE, AMAMOTO H. Bleeding esophageal ulcers caused by NSAIDs. Surg. Endosc., 1997 Feb, 11(2) : 143-6.

5. WILCOX CM, SCHWARTZ DA, CLARK WS. Esophageal ulceration in human immunodeficiency virus infection. Causes, response to therapy, and long-term outcome. Ann. Intern. Med., 1995 Jul 15, 123(2) : 143-9.

6. JOHNSON CD. Unusual complications of oesophageal ulcers. Postgrad. Med. J., 1988 Sep, 64(755) : 656-8.

7. RANATANEN TK, SIVHO EI, RASANEN JV, HYNNINEN M, SALO JA. Esophageal Ulcer as a Cause of Death: A Population-Based Study. Mortality of Esophageal Ulcer Disease. Digestion, 2015, 91(4) : 272-6. 